Pontifícia Universidade Catálica $_{\text {a }}$

Julio César Cuisano Egúsquiza

\title{
Avaliação Experimental de um Motor do Ciclo Diesel Operando no Modo Bicombustível: Diesel/Etanol e Diesel/Gás
}

Tese de Doutorado

Tese apresentada ao Programa de Pós-Graduação em Engenharia Mecânica da PUC-Rio como requisito parcial para obtenção do título de Doutor Engenharia Mecânica.

Orientador: Prof. Sergio Leal Braga Co-Orientador: Prof. Carlos Valois Maciel Braga 


\section{Julio César Cuisano Egúsquiza}

\section{Avaliação Experimental de um Motor do Ciclo Diesel Operando no Modo Bicombustível: Diesel/Etanol e Diesel/Gás}

Tese apresentada como requisito parcial para obtenção do grau de Doutor pelo Programa de Pós-Graduação em Engenharia Mecânica da PUC-Rio. Aprovada pela Comissão Examinadora abaixo assinada.

Prof. Sergio Leal Braga

Orientador

Departamento de Engenharia Mecânica - PUC-Rio

Prof. Carlos Valois Maciel Braga Co-Orientador

Departamento de Engenharia Mecânica - PUC-Rio

Prof. Marcos Sebastião de Paula Gomes Departamento de Engenharia Mecânica - PUC-Rio

Prof. José Alberto dos Reis Parise Departamento de Engenharia Mecânica - PUC-Rio

Prof. Fabrício José Pacheco Pujatti Universidade Federal de Minas Gerais

Prof. José Ricardo Sodré Pontifícia Universidade Católica de Minas Gerais

Prof. Edimilson Jesus de Oliveira CENPES/PETROBRAS

Prof. José Eugenio Leal

Coordenador Setorial do Centro Técnico Científico - PUC-Rio 
Todos os direitos reservados. É proibida a reprodução total ou parcial do trabalho sem autorização da universidade, do autor e do orientador.

\section{Julio César Cuisano Egúsquiza}

Graduou-se em Engenharia Mecânica de Fluidos pela Universidad Nacional Mayor de San Marcos, Lima-Perú, em 2000. Desde 2001 até 2004 atuou como engenheiro de projetos em diversas empresas peruanas, na área de energia e motores de combustão interna. Obteve o título de mestre em Engenharia Mecânica na PUC-Rio em 2006 (termociências experimental, com ênfase na redução das emissões em motores Diesel-gás). Atualmente é pesquisador e coordenador de projetos no LEV/PUC-Rio, estando envolvido no uso de combustíveis alternativos em motores de combustão interna.

Ficha Catalográfica

Cuisano Egúsquiza, Julio Cesar

Avaliação experimental de um motor do ciclo diesel operando no modo bicombustível: diesel / etanol e diesel / gás / Julio Cesar Cuisano Egúsquiza ; orientadores: Sergio Leal Braga, Carlos Valois Maciel Braga. -2011.

168 f. : il. (color.) ; $30 \mathrm{~cm}$

Tese (doutorado) - Pontifícia Universidade Católica do Rio de Janeiro, Departamento de Engenharia Mecânica, 2011. Inclui bibliografia

1. Engenharia mecânica - Teses. 2. Motor bicombustível. 3. Diesel-gás. 4. Diesel-etanol. 5. Combustão. 6. Emissões. I. Braga, Sergio Leal. II. Braga, Carlos Valois Maciel. III. Pontifícia Universidade Católica do Rio de Janeiro. Departamento de Engenharia Mecânica. IV. Título. 
Dedico este trabalho a minha amada esposa Zaida, meu amado filho Maurício e meus queridos pais Josefina e Maximiliano. 


\section{Agradecimentos}

Agradeço a Deus por tudo na minha vida. Por toda a força, luz e determinação na escolha da direção correta. Agradeço a Ele todas as vitórias e conquistas alcançadas.

Agradeço à minha família, meus pais, meus irmãos, meus sogros e cunhados pelo apoio e pela compreensão. Agradeço especialmente a minha amada esposa Zaida e meu amado filho Maurício que são o maior presente que Deus poderia ter me dado nesta vida. Por toda compreensão, apoio, sacrifício, incentivo, felicidade, carinho, dedicação e inspiração encontrada na minha querida família que sempre farão parte de cada luta e vitória.

Ao Professor Sergio Leal Braga, meu orientador e amigo, pela confiança em mim depositada. Agradeço por ter acreditado no meu potencial e por todas as oportunidades que me deu. Muito obrigado pelo acompanhamento, motivação, revisão e sugestões na redação do presente trabalho. Para mim foi um grande prazer poder trabalhar ao seu lado.

Ao Professor Carlos Valois Maciel Braga, meu co-orientador e também amigo, pelo qual tenho muita estima e admiração. Muito obrigado pelas idéias e pelas inúmeras conversas esclarecedoras e debates em torno do trabalho e pelas revisões no decorrer da redação do presente manuscrito.

Ao Departamento de Engenharia Mecânica da PUC-Rio, pelos auxílios concedidos e pelos ensinamentos.

Ao Instituto Tecnológico, ITUC/PUC-Rio, pelo suporte com a construção de equipamentos e a logística para a compra de insumos e instrumentação para a Sala de Testes de Motores do Laboratório de Engenharia Veicular, LEV/PUC-Rio. 
Aos meus amigos Pedro Oliveira Delbons, Laise Garcia e Gabriel Werpel Fernandes, que estiveram lado a lado comigo no desenvolvimento de grande parte deste trabalho realizando seu estágio no LEV/PUC-Rio. Por toda a dedicação e por não medir esforços no apoio das diversas tarefas relacionados à tese.

Aos colegas do LEV/PUC-Rio, Giovanni Calfa Neto e Ricardo Hernandez Pereira pelo companheirismo e continuado incentivo; Severino Wanderley e Nestor Corrêa Cotelo pelo suporte eletrônico e importantes contribuições; Fabrício Ferraz Gonçalves e Gilson Coutinho Pradanoff pelo auxilio na montagem do aparato experimental; Gerson Silveiro pela assistência na manutenção dos equipamentos.

Aos meus amigos do CENPES/PETROBRAS, Antônio Carlos Scardini Vilella e Guilherme Bastos Machado, pela troca de ideias e o suporte bibliográfico.

Ao, na época aluno de Iniciação Cientifica, Pedro Nickele Azevedo pelo auxilio nas medidas de desempenho e ao estagiário Leonardo Braga pelo auxilio nos desenhos.

Aos companheiros de pós-graduação, Luis Castillo, Miguel Mozo, Cesar Vera, Anthony Roque, Jose Alberto Aguilar Franco, Fernando Ferrari Filho, Gustavo Cezar, Fernando Zegarra pela amizade e pelos momentos de descontração na sala de estudos.

Aos meus amigos Luis Enrique Alva Huapaya, Roberto Quevedo Quispe, Habib Zambrano e Beatriz Mejia, David Maldonado e Martha Diaz, Carina Waller, Elizabeth Augusta, Marcia Coutinho e Jussara Novaes, pela amizade, apoio e momentos compartilhados.

Aos meus queridos amigos Aguinaldo, Marisa, Stephanie, Murilo e Isabella por acolher-nos como integrantes da família. Muito obrigado pelo apoio, convívio e pelo carinho. Que Deus ilumine essa linda família. 


\section{Resumo}

Egúsquiza, Julio César Cuisano; Braga, Sergio Leal; Braga, Carlos Valois Maciel. Avaliação Experimental de um Motor do Ciclo Diesel Operando no Modo Bicombustível: Diesel/Etanol e Diesel/Gás. Rio de Janeiro, 2011. 168p. Tese de Doutorado - Departamento de Engenharia Mecânica, Pontifícia Universidade Católica do Rio de Janeiro.

No presente trabalho, ensaios experimentais de um motor do ciclo Diesel consumindo etanol hidratado ou gás natural em substituição parcial ao óleo diesel, foram realizados. Os objetivos principais foram verificar as influências dos combustíveis alternativos e avaliar as técnicas do avanço da injeção do diesel e da restrição parcial do ar de admissão, em relação aos parâmetros característicos da combustão, desempenho e emissões. Com base nos dados do diagrama pressãoângulo de virabrequim, foi possível analisar alguns parâmetros característicos da combustão, tais como o início da combustão, a máxima taxa de elevação de pressão e o pico de pressão. Os parâmetros do desempenho e emissões do motor foram analisados através do rendimento térmico e as concentrações de monóxido de carbono, hidrocarbonetos, material particulado e óxidos de nitrogênio. Os resultados obtidos mostraram que as técnicas avaliadas no modo bicombustível junto com as elevadas taxas de substituição do óleo diesel favoreceram a melhor queima dos combustíveis alternativos, refletindo-se favoravelmente em menores emissões de CO e MP, além de um pequeno aumento no rendimento térmico do motor. No entanto, houve também um acréscimo nas emissões de $\mathrm{NO}_{\mathrm{X}} \mathrm{e}$, no caso específico do avanço da injeção, foi notado um maior ruído gerado pelo motor.

\section{Palavras-chave}

Motor bicombustível; diesel-gás; diesel-etanol; combustão; emissões. 


\section{Abstract}

Egúsquiza, Julio César Cuisano; Braga, Sergio Leal (Advisor); Braga, Carlos Valois Maciel. Experimental Investigation of a Diesel Cycle Engine Operating on Dual-Fuel Mode: Diesel / Ethanol and Diesel / Gas. Rio de Janeiro, 2011. 168p. DSc. Thesis - Departamento de Engenharia Mecânica, Pontifícia Universidade Católica do Rio de Janeiro.

In this report, experimental tests of a Diesel cycle engine running with hydrous ethanol or natural gas with partial substitution for diesel fuel were performed. The main objectives were to verify the influence of alternative fuels and evaluate the advancing of diesel injection timing and the air partial restriction, regarding the characteristic parameters of combustion, performance and emissions. Based on data from the pressure-crank angle diagram, it was possible to analyze some characteristic parameters of combustion, such as the start of combustion, the maximum rate of pressure rise and peak pressure. The parameters of the engine performance and emissions were analyzed through the thermal efficiency and the concentrations of carbon monoxide, hydrocarbons, particulate matter and nitrogen oxides. The results showed that the techniques evaluated in dual fuel mode with higher rates of substitution of diesel fuel favored a better burning of the alternative fuels, reflecting favorably in lower emissions of $\mathrm{CO}$ and $\mathrm{PM}$, and also in a small increase in the engine thermal efficiency. However, there was also an increase in $\mathrm{NO}_{\mathrm{X}}$ emissions and, in the specific case of the advanced injection timing, it was noted a louder noise generated by the engine.

\section{Keywords}

Dual fuel engine; diesel-gas; diesel-ethanol; combustion; emissions. 


\section{Sumário}

1 Introdução 19

1.1. Objetivos 23

1.2. Organização da Tese 23

2 Revisão da Literatura $\quad 25$

2.1. Uso de alcoóis em motores de ignição por compressão 26

2.1.1. Misturas álcool-óleo diesel 28

2.1.2. Emulsões álcool-óleo diesel 30

2.1.3. Injeção de álcool junto ao ar de admissão ("fumigação") 32

2.1.4. Injeção do álcool na câmara de combustão (dupla injeção) 38

2.1.5. Conversão do ciclo Diesel para o ciclo Otto ("ottolização") 40

2.1.6. Ativadores da ignição ("melhoradores") 41

2.2. Uso de combustíveis gasosos em motores de ignição por compressão 44

2.2.1. A operação do motor Diesel no modo bicombustível Diesel/gás 45

2.2.2. A combustão no modo Diesel/gás 47

2.2.3. Atraso da ignição no modo Diesel/gás 49

2.2.4. Combustão no modo Diesel/gás sob condições de baixas cargas 53

2.2.5. Emissão de gases de escape no modo Diesel/gás 56

2.2.6. Detonação no modo Diesel/gás 61

3 Aparato e Procedimento Experimental 64

3.1. Aparato experimental 64

3.2. Procedimento experimental $\quad 75$

4 Redução de Dados $\quad 78$

4.1. Análise das incertezas experimentais 84

5 Resultados e Discussões $\quad 88$

5.1. Resultados Diesel/etanol 89 
5.1.1. Efeitos do etanol hidratado sobre as características da combustão

5.1.2. Efeitos do avanço da injeção do óleo diesel sobre as características da combustão no modo Diesel/etanol

5.1.3. Efeitos do etanol hidratado e do avanço da injeção do óleo diesel relacionados aos parâmetros de desempenho e emissões 101 5.2. Resultados Diesel/gás

5.2.1. Efeitos do gás natural sobre as características da combustão

5.2.2. Efeitos do avanço da injeção do diesel e da restrição parcial do ar de admissão sobre as características da combustão no modo Diesel/gás

5.2.3. Efeitos do avanço da injeção do diesel e da restrição parcial do ar de admissão relacionados aos parâmetros de desempenho e emissões

6 Conclusões e Recomendações

Referências Bibliográficas

Apêndice I - Propriedades termofísicas

Apêndice II - Vazão de ar úmido 151

Apêndice III - Relações estequiométricas ar - combustível

154

Apêndice IV - Pontos experimentais 158

Apêndice V - Restrição parcial do ar no modo Diesel / etanol 


\section{Lista de Figuras}

Figura 1 - Representação esquemática dos diferentes componentes da taxa de liberação de energia da combustão em um motor Diesel/gás (Karim, 2003).

Figura 2 - Variações do ponto de ignição com a razão de equivalência total para diferentes combustíveis, mantendo a quantidade do piloto constante. A correspondente operação diesel é também mostrada (Karim e Burn, 1980).

Figura 3 - Consumo específico dos combustíveis (diesel e gás natural) variando com as taxas de substituição e as cargas do motor (Egúsquiza et al., 2009).

Figura 4 - Emissões de hidrocarbonetos não queimados variando com a taxa de substituição (diesel por gás natural) e as cargas do motor (Egúsquiza et al., 2009).

Figura 5 - Emissão de monóxido de carbono variando com a taxa de substituição (diesel por gás natural) e as cargas do motor (Egúsquiza et al., 2009).

Figura 6 - Emissões óxidos de nitrogênio variando com a taxa de substituição (diesel por gás natural) e as cargas do motor (Egúsquiza et al., 2009).

Figura 7 - Emissões de material particulado variando com a taxa de substituição (diesel por gás natural) e as cargas do motor (Egúsquiza, 2006).

Figura 8 - Esquema do aparato experimental.

Figura 9 - Vistas do motor ensaiado - modelo MWM 4.10 TCA.

Figura 10 - Esquema da bomba distribuidora BOSCH VE utilizada pelo motor ensaiado (Bosch, 2003).

Figura 11 - Ajuste do avanço "estático" da injeção do óleo diesel no motor ensaiado.

Figura 12 - Vistas do dinamômetro de bancada e motor em montagem para testes.

Figura 13 - Medidor de tipo laminar utilizado nas medidas do consumo de ar.

Figura 14 - Instalação da válvula manual usada na restrição do ar.

Figura 15 - Balança de combustível utilizada na medição do consumo de óleo diesel.

Figura 16 - Medidores de vazão tipo Coriolis e reguladores de pressão (lado B) utilizados no controle e medição da vazão de etanol e gás natural. 
Figura 17 - Smoke Meter e ponto de amostragem no escapamento.

Figura 18 - Equipamento portátil Testo e ponto de amostragem no escapamento.

Figura 19 - Esquema experimental para medição da pressão no cilindro.

Figura 20 - Instrumentação usada para medição da pressão no cilindro.

Figura 21 - Início da combustão obtido através do expoente politrópico da compressão e dos valores picos da segunda e terceira derivada da pressão.

Figura 22 - Variação das curvas de pressão com a substituição Diesel/etanol e a carga do motor (RPM: 1.800); injeção do diesel: $P O=9^{\circ}$ APMS.

Figura 23 - Coeficiente politrópico de compressão versus taxa de substituição, carga e rotação do motor; ponto de injeção do diesel: $P O=9^{\circ}$ APMS.

Figura 24 - Início da combustão variando com a substituição

Diesel/etanol, carga e rotação do motor; ponto de injeção do diesel: $P O=9^{\circ}$ APMS.

Figura 25 - Taxa máxima do aumento da pressão no cilindro variando com a substituição Diesel/etanol, carga e rotação; injeção do diesel: $P O=9^{\circ}$ APMS.

Figura 26 - Pressão máxima no cilindro variando com a substituição Diesel/etanol, carga e rotação do motor; injeção do diesel: $P O=9^{\circ}$ APMS .

Figura 27 - Ângulo da pressão máxima variando com a substituição Diesel/etanol, carga e rotação do motor; ponto de injeção do diesel: $P O=9^{\circ}$ APMS .

Figura 28 - Curvas de pressão no cilindro variando com a taxa de substituição Diesel/etanol, pontos de injeção de diesel e carga do motor (RPM: 1.800).

Figura 29 - Início da combustão variando com a taxa de substituição Diesel/etanol, ponto de injeção de diesel e carga do motor (RPM: 1.800).

Figura 30 - Taxa máxima de aumento da pressão variando com a substituição Diesel/etanol, ponto de injeção de diesel e carga do motor (RPM: 1.800).

Figura 31 - Pressão máxima no cilindro variando com a taxa de substituição Diesel/etanol, ponto de injeção de diesel e carga do motor (RPM: 1.800).

Figura 32 - Ângulo da pressão máxima variando com a substituição Diesel/etanol, ponto de injeção de diesel e carga do motor (RPM: 1.800). 
Figura 33 - Rendimento térmico variando com a taxa de substituição Diesel/etanol, ponto de injeção de diesel e carga do motor (RPM: 1.800).

Figura 34 - Emissões de óxidos de nitrogênio variando com a taxa de substituição Diesel/etanol, ponto de injeção de diesel e carga do motor (RPM: 1.800).

Figura 35 - Emissões de hidrocarbonetos variando com a taxa de substituição Diesel/etanol, ponto de injeção de diesel e carga do motor (RPM: 1.800).

Figura 36 - Emissão de monóxido de carbono variando com a taxa de substituição Diesel/etanol, ponto de injeção de diesel e carga do motor (RPM: 1.800).

Figura 37 - Emissão de material particulado variando com a taxa de substituição Diesel/etanol, ponto de injeção de diesel e carga do motor (RPM: 1.800).

Figura 38 - Variações das curvas de pressão com a taxa de substituição Diesel/gás e a carga do motor (RPM: 1.800); ponto de injeção do diesel: $P O=9^{\circ} A P M S$.

Figura 39 - Coeficiente politrópico de compressão versus taxa de substituição, carga e rotação do motor; ponto de injeção do diesel: $P O=9^{\circ} A P M S$.

Figura 40 - Início da combustão variando com a taxa de substituição Diesel/gás, a carga e a rotação do motor; ponto de injeção do diesel: $P O=9^{\circ}$ APMS.

Figura 41 - Início da combustão variando com a razão de equivalência total, a carga e a rotação do motor; ponto de injeção do diesel: $P O=9^{\circ} A P M S$.

Figura 42 - Taxa máxima de aumento de pressão no cilindro variando com a substituição Diesel/gás, a carga e a rotação; injeção do diesel: $P O=9^{\circ} A P M S$.

Figura 43 - Pressão máxima no cilindro variando com a substituição Diesel/gás, a carga e a rotação do motor; ponto de injeção do diesel: $P 0=9^{\circ}$ APMS.

Figura 44 - Ângulo da pressão máxima variando com a substituição Diesel/gás, a carga e a rotação do motor; ponto de injeção do diesel: $P O=9^{\circ} A P M S$.

Figura 45 - Pressão indicada variando com o ponto de injeção de diesel e a restrição do ar de admissão; pme= 1,25 e 0,94 MPa, $1.800 \mathrm{rpm}, \mathrm{TS}=50 \%$.

Figura 46 - Início da combustão versus carga, taxa de substituição Diesel/gás, ponto de injeção do diesel e restrição do ar de admissão (RPM: 1.800). 
Figura 47 - Taxa máxima do aumento da pressão versus carga, substituição Diesel/gás, ponto de injeção diesel e restrição do ar de admissão (RPM: 1.800).

Figura 48 - Pressão máxima versus carga, taxa de substituição Diesel/gás, ponto de injeção diesel e restrição do ar de admissão (RPM: 1.800).

Figura 49 - Ângulo da pressão máxima versus carga, taxa de substituição Diesel/gás, ponto de injeção diesel e restrição do ar de admissão (RPM: 1.800).

Figura 50 - Rendimento térmico versus carga, taxa de substituição Diesel/gás, ponto de injeção do diesel e restrição do ar de admissão (RPM: 1.800).

Figura 51 - Emissões de monóxido de carbono versus carga, taxa de substituição Diesel/gás, ponto de injeção do diesel e restrição do ar de admissão (RPM: 1.800).

Figura 52 - Emissões de óxidos de nitrogênio versus carga, taxa de substituição Diesel/gás, ponto de injeção do diesel e restrição do ar de admissão (RPM: 1.800).

Figura 53 - Emissões de material particulado versus carga, taxa de substituição Diesel/gás, ponto de injeção do diesel e restrição do ar de admissão (RPM: 1.800).

Figura AV: Rendimento térmico e emissões de $\mathrm{HC}$, $\mathrm{CO}$ e $\mathrm{NO}_{\mathrm{x}}$ variando com a taxa de substituição Diesel/etanol e a restrição do ar; pme $=0,36 \mathrm{MPa} ; \mathrm{RPM}=1.800$; ponto de injeção do diesel: $P O=9^{\circ} A P M S$. 


\section{Lista de Tabelas}

Tabela 1 - Principais características técnicas do motor MWM 4.10 TCA.

Tabela 2 - Valores de avanço da injeção utilizados nos ensaios.

Tabela Al - Propriedades termofísicas do ar, metano e etanol

$\left(15^{\circ} \mathrm{C}\right.$ e $\left.1 \mathrm{~atm}\right)$.

150

Tabela Alll - Composição média do gás natural fornecido pela CEG.

Tabela AIV.I - Resultados Diesel/Etanol.

Tabela AIV.II - Resultados Diesel/Gás. 


\section{Nomenclatura}

\begin{tabular}{|c|c|}
\hline APMS & Antes do ponto morto superior \\
\hline$b_{a r, s}$ & Constante para cálculo da viscosidade dinâmica do ar seco \\
\hline C & Carbono \\
\hline $\mathrm{CO}$ & Monoxido de carbono \\
\hline $\mathrm{CO}_{2}$ & Dioxido de Carbono \\
\hline $\mathrm{HC}$ & Hidrocarbonetos não queimados \\
\hline$I$ & Incerteza relativa \\
\hline k & Relação de calores específicos \\
\hline$\dot{m}$ & Vazão mássica \\
\hline M & Massa molar \\
\hline MP & Material particulado \\
\hline$n$ & Velocidade angular ou expoente politrópico \\
\hline $\mathrm{N}_{2}$ & Nitrogênio \\
\hline NO & Óxido nítrico \\
\hline $\mathrm{NO}_{2}$ & Dioxido de nitrogênio \\
\hline $\mathrm{NO}_{x}$ & Óxidos de nitrogênio \\
\hline NC & Número de cetano \\
\hline $\mathrm{O}_{2}$ & Oxigênio \\
\hline $\mathrm{P}$ & Potência efetiva \\
\hline $\mathrm{P}_{\mathrm{ar}}$ & Pressão barometrica \\
\hline $\mathrm{PCl}$ & Poder calorífico inferior \\
\hline $\begin{array}{l}\mathrm{p}_{\text {sat }} \\
\text { ambiente }\end{array}$ & Pressão de saturação do vapor de água à temperatura \\
\hline PMS & Ponto morto superior \\
\hline PMI & Ponto morto inferior \\
\hline pme & Pressão média efetiva \\
\hline ppm & Partes por milhão \\
\hline P0 & Avanço original da injeção do diesel ( $9^{\circ}$ APMS) \\
\hline $\mathrm{P} 1$ & Avanço da injeção do diesel equivalente a $10^{\circ} \mathrm{APMS}$ \\
\hline P2 & Avanço da injeção do diesel equivalente a $11^{\circ} \mathrm{APMS}$ \\
\hline
\end{tabular}


P4 Avanço da injeção do diesel equivalente a $13^{\circ}$ APMS

$\mathrm{R} \quad$ Constante universal dos gases

RA Restrição do ar

RET Razão de equivalência total

RPM Rotações por minuto

$\mathrm{SO}_{\mathrm{x}} \quad$ Óxido de enxofre

$S_{\mathrm{ar}, \mathrm{s}} \quad$ Constante para cálculo da viscosidade dinâmica do ar seco

$T \quad$ Torque efetivo

$\mathrm{T}_{\mathrm{amb}} \quad$ Temperatura ambiente

TS Taxa de substituição

$\mathrm{U}_{\mathrm{r}} \quad$ Umidade relativa

$\mathrm{V}_{\mathrm{d}} \quad$ Volume deslocado por ciclo

$\dot{V} \quad$ Vazão volumetrica

Y Fração molar

w Umidade absoluta

$\mathrm{W}_{\mathrm{c}} \quad$ Trabalho desenvolvido por ciclo

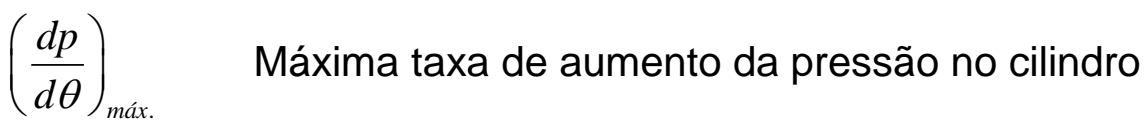

$\frac{\partial Q_{c}}{\partial \theta} \quad$ Taxa de calor liberado pelo combustível 


\title{
Subscritos e Letras Gregas
}

\author{
ar,u $\quad$ Ar úmido \\ ar,s $\quad$ Ar seco \\ d Relativo à vazão mássica do combustível diesel. \\ d,o Relativo ao consumo de diesel original \\ e Etanol \\ g Gás natural \\ $\rho \quad$ Densidade \\ $r \quad$ Relativo às condições atmosféricas de referência \\ $\Delta P \quad$ Diferencial de pressão \\ $\mu \quad$ Viscosidade dinãmica \\ $\eta_{T} \quad$ Rendimento termico efetivo \\ $\phi \quad$ Razão de equivalência \\ $\theta_{I C} \quad$ Ângulo correspondente ao início da combustão
}

